\title{
SCNS.02. Evaluation of xanthine oxidase enzyme inhibition in a model of depressive- like behavior induced by LPS in mice
}

TOMAZ, V.S. ${ }^{1}$, CORDEIRO, R.C. ${ }^{2}$, MONTEIRO, I.O3 ${ }^{3}$ MACEDO, D. ${ }^{3}$

${ }_{1}^{1}$ Departamento de Microbiologia Médica, Faculdade de Medicina, Universidade Federal do Ceará. 2 Departamento de Medicina Clínica, Faculdade de Medicina, Universidade Federal do Ceará.

3 Departamento de Fisiologia e Farmacologia, Faculdade de Medicina, Universidade Federal do Ceará.

Introduction: The participation of nitric oxide (NO) in the pathophysiology of depression has been extensively studied, suggesting an important role of this gas in depression. Several inhibitors of nitric oxide synthase (NOS) were considered potent antidepressant agents. The xanthine oxidase $(\mathrm{XO})$ in hypoxic conditions and in the presence of NADH is able to catalyze organic nitrates into NO, thereby the inhibition of this enzyme reduces the availability of $\mathrm{NO}$ in organisms. Based on this hypothesis we evaluated the inhibition of $\mathrm{XO}$ as an indirect way to observe the role of $\mathrm{NO}$ in an experimental model of depressive-like behavior. Objectives: To evaluate the effects of an inhibition of xanthine oxidase in a model of depressive-like behavior induced by LPS in mice. Methods: Adult male Swiss mice (20-30g, $\mathrm{n}=6-8$ per group) were treated with allopurinol (100 mg / kg, ip), imipramine (10 mg kg , ip), LPS (0.5 mg / kg, ip) or saline. Animals were divided into four groups, two were pretreated with allopurinol or Imipramine 30 minutes before LPS administration, one received only the dose of LPS and the control group received only saline. Animals were submitted to evaluation of the depressive-like behavior through the forced swimming test, $24 \mathrm{~h}$ after LPS administration a time-point after LPS administration related to the occurrence of depressive-like behavior. After behavioral testing, animals were sacrificed by cervical dislocation and their brain areas: Pre-frontal Cortex (PF), Striatum (ST) and Hippocampus (HP) were separated and stored at $-80^{\circ} \mathrm{C}$ for later measurement of nitrite levels by method according to Gries. The statistical analysis was performed by ANOVA followed by Tukey's test, considering significant $p<0.05$. The project was approved by the Ethics Committee on Animal Research of UFC. Results: When compared to control group, animals that received only LPS increased immobility time. In pre-treatment with imipramine, immobility time remained similar to control group, confirming imipramine's antidepressant activity, a drug used in clinical medicine for the treatment of depression. Allopurinol pretreated animals presented results similar to those animals that received only LPS (control: $68.71 \pm 17.05$, LPS: $151.30 \pm 9.58$; Imipramine: $50.20 \pm 3.00$; Allopurinol: $170.00 \pm$ 23.63). Regarding the dosage of nitrite, significant increase was not observed in this parameter in PF, but in HP was observed a significant increase in animals pretreated with allopurinol when compared to the group that received only LPS (PF- LPS: $50.90 \pm 9.67$, Control: $126.40 \pm 71.90$, Imipramine: $26.82 \pm 1.59$; Allopurinol: $151.50 \pm 51.17$; HP- LPS: $86.23 \pm 40.84$, Control: $299.70 \pm 73.89$, Imipramine: $26.82 \pm 1.59$; Allopurinol: $303.80 \pm$ 71.70; ST: LPS: $145.80 \pm 64.93$, Control: $184.1 \pm 19.89$, Imipramine: $26.82 \pm 1.59$; Allopurinol: $212.80 \pm 11.67)$. Conclusions: LPS induced depressive-like behavior as verified in the forced swim test. When treated with allopurinol at $100 \mathrm{mg} / \mathrm{kg}$, an inhibitor of the enzyme XO, it was observed that there is a significant increase in immobility time, which suggests that the inhibition of the enzyme is able to enhance the depressive-like effects induced by LPS.

Keywords: Depression, LPS, Xanthine Oxidase, Allopurinol.

TOMAZ, V.S.; CORDEIRO, R.C.; MONTEIRO, I.O; MACEDO, D. 2013. Evaluation of xanthine oxidase enzyme inhibition in a model of depressivelike behavior induced by LPS in mice, p.12. In: Oriá, Reinaldo Barreto; Andrade, Geanne Matos de; Bruin, Veralice Meireles S. de. I International Symposium in Neuroscience Meeting [Blucher Neuroscience Proceedings n.1 v.1]. São Paulo: Blucher, 2014, http://dx.doi.org/10.5151/isnm-sine7 